Pacific Journal of Mathematics

BEST SIMULTANEOUS DIOPHANTINE APPROXIMATIONS. II.
BEHAVIOR OF CONSECUTIVE BEST APPROXIMATIONS 


\title{
BEST SIMULTANEOUS DIOPHANTINE APPROXIMATIONS II. BEHAVIOR OF CONSECUTIVE BEST APPROXIMATIONS
}

\author{
J. C. LAGARIAS
}

It is well-known that the best Diophantine approximations to a single real number $\theta$ are exactly the convergents of the continued fraction expansion of $\theta$. The properties of one-dimensional best approximations that make this true are shown not to hold in general for best simultaneous Diophantine approximations to $\alpha \in R^{n}$ when $n \geqq 2$. They do hold in a weak form for all badly approximate vectors $\alpha \in \boldsymbol{R}^{n}$.

1. Introduction. In this paper we study properties of the set of best simultaneous Diophantine approximations to a vector $\alpha \in \boldsymbol{R}^{n}$ with respect to a norm $\|\cdot\|$ on $\boldsymbol{R}^{n}$. We recall the basic definitions. For $\alpha=\left(\alpha_{1}, \cdots, \alpha_{N}\right)$ and a denominator $q>0$ the quantity

$$
\delta_{q}=\delta_{q}(\alpha)=\underset{\left(p_{1}, \cdots, p_{n}\right) \in \mathbf{Z}^{n}}{\operatorname{MIN}}\left\|\left(q \alpha_{1}-p_{1}, \cdots, q \alpha_{n}-p_{n}\right)\right\|
$$

measures the degree of approximation to $\alpha$ possible by rationals of denominator $q$. The best simultaneous approximation denominators (BSAD's) $q_{k}=q_{k}(\alpha)$ to $\alpha$ with respect to the norm $\|\cdot\|$ are inductively defined by $q_{1}=1$ and by $q_{k}$ being the least positive integer such that $\delta_{q_{k}}<\delta_{q_{k-1}}$. The corresponding best simultaneous approximations $\left(\mathrm{BSA}^{\prime} \mathrm{s}\right) \boldsymbol{v}_{k}$ are the integer vectors

$$
\boldsymbol{v}_{k}=\boldsymbol{v}_{k}(\alpha)=\left(q_{k}, p_{k, 1}, \cdots, p_{k, n}\right)
$$

in $\boldsymbol{Z}^{n+1}$ where $\left(p_{k, 1}, \cdots, p_{k, n}\right)$ attains the minimum in (1.1) for $q_{k}$. (In case the minimum in (1.1) for a BSAD is attained by more than one such $\boldsymbol{v} \in \mathbf{Z}^{n+1}$, we select one such in (1.2) arbitrarily. There are only finitely many possible BSAD's for which this may happen, c.f. Proposition 2.1. In general we call any vector $\boldsymbol{v}=\left(q, p_{1}, \cdots, p_{n}\right)$ minimizing (1.1) an approximation vector and

$$
\boldsymbol{R}(\boldsymbol{v})=\left(q \alpha_{1}-p_{1}, \cdots, q \alpha_{n}-p_{n}\right)
$$

its approximation remainder vector.

It is well-known that the best approximations to a single real number $\theta$ are exactly the convergents of the continued fraction expansion of $\theta$ (Lang [6], p. 10). The following properties of these (one-dimensional) best approximations form the basis of the continued fraction algorithm. 
(i) The determinants

$$
D_{k}=\left|\begin{array}{c}
\boldsymbol{v}_{k} \\
\boldsymbol{v}_{k+1}
\end{array}\right|=\left|\begin{array}{cc}
q_{k} & q_{k} \\
q_{k+1} & p_{k+1}
\end{array}\right|= \pm 1
$$
then

(ii) If $\boldsymbol{v}_{k-1}, \boldsymbol{v}_{k}$ are two successive best approximation vectors,

$$
\boldsymbol{v}_{k+1}=a \boldsymbol{v}_{k}+\boldsymbol{v}_{k-1}
$$

for some positive integer $a$.

In this paper we consider to what extent analogues of these properties hold for best simultaneous approximations in higher dimensions.

Higher-dimensional analogues of property (i) involve the $N+1$ by $N+1$ kth best approximation matrix defined by

$$
\boldsymbol{M}_{k}=\boldsymbol{M}_{k}(\alpha,\|\cdot\|)=\left[\begin{array}{c}
\boldsymbol{v}_{k} \\
\boldsymbol{v}_{k+1} \\
\boldsymbol{v}_{k+n}
\end{array}\right]=\left[\begin{array}{llll}
q_{k} & p_{k, 1} & \cdots & p_{k+n} \\
q_{k+1} & p_{k+1,1} & & p_{k+1, n} \\
\vdots & & & \\
q_{k+n} & p_{k+n, 1} & \cdots & p_{k+n, n}
\end{array}\right]
$$

and the kth best approximation determinant defined by

$$
D_{k}=\operatorname{det} \boldsymbol{M}_{k} \text {. }
$$

In $\S 3$ we show that there is a two-dimensional analogue of property (ii), which is related to the case that three consecutive best approximation vectors $\boldsymbol{v}_{k}, \boldsymbol{v}_{k+1}, \boldsymbol{v}_{k+2}$ are linearly dependent, i.e., when $D_{k}=0$.

THEOREM 1.1. For any $\alpha \in \boldsymbol{R}^{2}-\boldsymbol{Q}^{2}$ and any norm $\|\cdot\|$ on $\boldsymbol{R}^{2}$, there is a $k_{0}=k_{0}(\|\cdot\|)$ such that for $k \geqq k_{0}$ the following are equivalent.

(i) $D_{k}=0$.

(ii) $\boldsymbol{v}_{k}=a \boldsymbol{v}_{k-1}+\boldsymbol{v}_{k-2}$ for some positive integer $a$.

For the sup norm $\|\cdot\|_{s}$ we may take $k_{0}=1$.

The absence of an exact higher-dimensional analogue of the continued fraction algorithm is reflected in the failure of property (i) in all higher dimensions. In $\S 4$ we prove the following result concerning zero determinants.

THEOREM 1.2. For any given norm $\|\cdot\|$ on $\boldsymbol{R}^{n}$ with $n \geqq 2$ there exists an $\alpha \in \boldsymbol{R}^{n}$ with $\operatorname{dim}_{Q}\left[1, \alpha_{1}, \cdots, \alpha_{n}\right]=n+1$ such that for any positive integer $L$ there exists an integer $k$ (depending on $L$ ) such that the best approximation determinants of $\alpha$ with respect to $\|\cdot\|$ have the property 


$$
D_{k}=D_{k+1}=\cdots=D_{k+L}=0 .
$$

The proof of Theorem 1.2 actually shows in addition that we can force the corresponding best approximation matrices $\boldsymbol{M}_{k}, \boldsymbol{M}_{k+1}$, $\cdots, \boldsymbol{M}_{k+L}$ in (1.8) to all have rank exactly 2 . Concerning nonzero determinants, we show in the two-dimensional case for the sup norm that arbitrarily large determinants can occur.

THEOREM 1.3. Let \|\|$_{s}$ be the sup norm on $\boldsymbol{R}^{2}$. There exist $\alpha \in \boldsymbol{R}^{2}$ having the property that for any positive integer $L$ there exists

(i) an integer $k$ such that

$$
D_{k}=D_{k+1}=\cdots=D_{k+L}=0 .
$$

(ii) an integer $m$ such that

$$
\left|D_{k}\right|>L \text {. }
$$

Theorems 1.2 and 1.3 show that higher-dimensional analogues of the continued fraction algorithm must include other approximations than just the best simultaneous approximations with respect to a fixed norm $\|\cdot\|$. General discussions of multi-dimensional continued fraction algorithms and their properties are given in Brentjes [1] and Szekeres [8].

In $\S 5$ we consider the behavior of best simultaneous approximations to badly approximable vectors. A vector $\alpha$ in $\boldsymbol{R}^{N}$ is said to be badly approximable with approximation constant $C$ if there are only finitely many solutions to the inequality

$$
\underset{1 \leqq i \leqq n}{\operatorname{MAX}}\left|q \alpha_{i}-p_{i}\right|<C|q|^{-1 / n} .
$$

The main results of $\S 5$ show that badly approximable vectors cannot exhibit the pathological behavior of the vectors $\alpha$ constructed in Theorems 1.2 and 1.3. Applied to the two-dimensional case with the sup norm we obtain the following result.

THEOREM 1.4. Let $\alpha$ be a badly approximable vector in $\boldsymbol{R}^{2}$ with approximation constant $C$. For the sup norm $\|\cdot\|_{s}$ on $\boldsymbol{R}^{2}$ there are only finitely many solutions $k$ to both of:

(i) $\left|D_{k}\right|>L$ with $L=12 C^{-4}$.

(ii) $D_{k}=D_{k+1}=\cdots=D_{k+L}=0$ with

$$
L=[5+10|\log C|] \text {. }
$$

More general theorems are stated in $\S 5$.

Certain of the results of this paper were announced in [4], which contains relevant numerical examples. 
2. Preliminaries. We collect here some preliminary results about norms and best approximations.

The first fact is that an arbitrary norm $\|\cdot\|$ on $\boldsymbol{R}^{n}$ is compatible with the sup norm $\|\cdot\|_{s}$ on $\boldsymbol{R}^{n}$

$$
\|\boldsymbol{x}\|_{s}=\operatorname{MAX}_{1 \leqq i \leqq n}\left|x_{i}\right|
$$

in the sense that there are positive constants $c_{1}$ and $c_{2}$ (depending on $\|\cdot\|)$ such that

$$
c_{1}\|\boldsymbol{x}\| \leqq\|\boldsymbol{x}\|_{s} \leqq c_{2}\|\boldsymbol{x}\| \text {. }
$$

The norms $\|\cdot\|$ and $\lambda\|\cdot\|$ for $\lambda>0$ determine the same sets of best approximation vectors. Consequently we may deal without loss of generality in the rest of this paper with scaled norms having the properties

(i ) $\boldsymbol{x} \in \mathbf{Z}^{n}$ and $\boldsymbol{x} \neq 0=>\|\boldsymbol{x}\| \geqq 1$.

(ii) There is an $\boldsymbol{x} \in \mathbf{Z}^{n}$ with $\|\boldsymbol{x}\|=1$.

The Euclidean and sup norms are scaled norms.

We also recall Dirichlet's theorem.

DIRICHLET'S THEOREM. For any $\alpha \in \boldsymbol{R}^{n}$ and any $M$ there is a denominator $q \leqq M$ and integers $p_{i}$ such that

$$
\underset{1 \leqq i \leqq M}{\operatorname{MAX}}\left|q \alpha_{i}-p_{i}\right|<\left(\left[M^{-1 / n}\right]\right)^{-1}
$$

Dirichlet's theorem implies that for sup norm best simultaneous approximation denominators $q$ to a given $\alpha$,

$$
\delta_{q}(\alpha)<q^{-1 / n} \text {. }
$$

We also recall ([5], Lemma 2.1) a simple result which shows that best approximation vectors are well-defined except possibly for a few small denominators.

Proposition 2.1. (i) For any norm $\|\cdot\|$ on $\boldsymbol{R}^{n}$ and any $\varepsilon>0$ there is a bound $k_{0}=k_{0}(\|\cdot\|, \varepsilon)$ such that

$$
\delta_{q}(\alpha)<\varepsilon
$$

holds for all $\alpha \in \boldsymbol{R}^{n}$ and all best approximation denominators $q$ to $\alpha$ with respect to $\|\cdot\|$ for which $q>k_{0}$.

(ii) For a scaled norm $\|\cdot\|$, a vector $\alpha \in \boldsymbol{R}^{n}$ and any denominator $q$ for which

$$
\delta_{q}(\alpha)<\frac{1}{2}
$$


there is a unique approximation vector $v=\left(q, p_{1}, \cdots, p_{n}\right)$ with denominator $q$.

3. $Z$-linear dependence relations. As a first step in analyzing the behavior of consecutive best approximation vectors, we consider the restrictions that $Z$-linear dependencies among $\left[1, \alpha_{1}, \cdots, \alpha_{n}\right]$ impose on the best approximation vectors to $\alpha$, and we show there are restrictions on the form of $Z$-linear dependencies that may occur among consecutive best approximation vectors.

We use a result of Mack [7] to show that any $Z$-linear dependence among $\left[1, \alpha_{1}, \cdots, \alpha_{n}\right]$ is satisfied by all sufficiently large best approximation vectors $\boldsymbol{v}\left(q_{k}\right)$. This is a property not only of best approximation vectors, but of all sufficiently good approximations. We say a vector $v=\left(q, p_{1}, \cdots, p_{n}\right)$ is a $\lambda$-good approximation provided

$$
\operatorname{MAX}_{1 \leqq i \leqq n}\left|q \alpha_{i}-p_{i}\right|<\lambda|q|^{-1 / n}
$$

Dirichlet's theorem implies that all sup norm best approximation vectors are 1-good approximations. Then (2.1) implies that all best approximation vectors to a norm $\|\cdot\|$ are $\lambda$-good with $\lambda=c_{1}^{-1}$.

THEOREM 3.1. (Mack). Suppose the components of $\alpha \in \boldsymbol{R}^{n}$ satisfy the Z-linear dependence relation

$$
a_{0}=\sum_{i=1}^{n} a_{i} \alpha_{i}
$$

where $\alpha_{i} \in Z$. Set

$$
A=\operatorname{MAX}_{0 \leqq i \leqq n}\left|a_{i}\right| \text {. }
$$

If $\left(q, p_{1}, \cdots, p_{n}\right)$ is a $\lambda$-good approximation to $\alpha$ for which

$$
q>(n A \lambda)^{n}
$$

then

$$
a_{0} q=\prod_{i=1}^{n} a_{i} p_{i}
$$

Proof. Let $\left(q, p_{1}, \cdots, p_{n}\right)$ be an approximation vector satisfying (3.4) and find $k \in \boldsymbol{Z}$ so that

$$
\sum_{i=1}^{n} a_{i} p_{i}=a_{0} q+k .
$$

Subtracting $q$ times (3.2) from this equation yields 


$$
\sum_{i=1}^{n} a_{i}\left(p_{i}-q \alpha_{i}\right)=k \text {. }
$$

Using (3.1), (3.3) we have the bound

$$
\left|\sum_{i=1}^{n} a_{i}\left(p_{i}-q \alpha_{i}\right)\right| \leqq n A \lambda q^{-1 / n} .
$$

The hypothesis (3.4) then gives

$$
\left|\sum_{i=1}^{n} a_{i}\left(p_{i}-q \alpha_{i}\right)\right|<1 \text {. }
$$

Since $k \in Z$ in (3.6) this forces $k=0$, establishing (3.5).

CoRollaRY 3.2. Let $\operatorname{dim}_{Q}\left[1, \alpha_{1}, \cdots, \alpha_{n}\right]=r$, so that the $\boldsymbol{Z}^{n+1}$ module $R$ of $Z$-linear dependence relations among $\left[1, \alpha_{1}, \cdots, \alpha_{n}\right]$ has rank $n+1-r$. Let $r_{1}, \cdots, r_{n+1-r}$ be a $Z$-basis of $R$ (which exists because $R$ is a subgroup of the free abelian group $Z^{n+1}$ ) and write

$$
\boldsymbol{r}_{i}=\left(a_{i 0}, a_{i 1}, \cdots, a_{i n}\right) \text {. }
$$

Set

$$
A=\operatorname{MAX}_{i, j}\left|\alpha_{i j}\right| \text {. }
$$

If $\boldsymbol{w}=\left(q, p_{1}, \cdots, p_{n}\right)$ is a $\lambda$-good approximation with

$$
q>(n A \lambda)^{n}
$$

then $\boldsymbol{w}$ satisfies the entire module of relations $R$.

CoROLlaRy 3.3. If $\operatorname{dim}_{Q}\left[1, \alpha_{1}, \cdots, \alpha_{n}\right]=r$ with $r \geqq 2$ then for any norm $\|\cdot\|$ on $\boldsymbol{R}^{n}$ there is a $k_{0}=k_{0}(\alpha,\|\cdot\|)$ such that for all $k>k_{0}$, the best approximation matrices $M_{k}$ have

$$
\operatorname{rank}_{\boldsymbol{R}} M_{k} \leqq \boldsymbol{r} .
$$

Corollary 3.3 allows the possibility that for $r \geqq 3$ there can be infinitely many $k$ such that $\operatorname{rank}_{R} M_{k}<r$ for a given $\alpha$. We believe the converse of Corollary 3.3 is true, and state this as a conjecture.

CONJECTURE 3.4. When $\alpha \in \boldsymbol{R}^{n}-\boldsymbol{Q}^{n}$, then the following are equivalent.

(i) $\operatorname{dim}_{Q}\left[1, \alpha_{1}, \cdots, \alpha_{n}\right] \leqq r$.

(ii) There is a $k_{0}=k_{0}(\alpha,\|\cdot\|)$ such that for $k \geqq k_{0}$, 


$$
\operatorname{rank}_{R} M_{k} \leqq r
$$

We will prove this conjecture when $r=2$.

THEOREM 3.5. When $\alpha \in \boldsymbol{R}^{n}-\boldsymbol{Q}^{n}$, the following are equivalent.

(i ) $\operatorname{dim}_{Q}\left[1, \alpha_{1}, \cdots, \alpha_{n}\right] \leqq 2$.

(ii) There is a $k_{0}=(\alpha,\|\cdot\|)$ such that for $k \geqq k_{0}$,

$$
\operatorname{rank}_{\mathbf{R}} M_{k} \leqq 2 \text {. }
$$

REMARK. Equality must hold in both inequalities in (i) and (ii) above. This is true for (i) because $\alpha \in \boldsymbol{R}^{n}-\boldsymbol{Q}^{n}$. For (ii) this is true because two consecutive best approximation vectors $\boldsymbol{v}_{k}, \boldsymbol{v}_{k+1}$ are linearly independent over $\boldsymbol{R}$. To see this, if $\boldsymbol{v}_{k+1}=\lambda \boldsymbol{v}_{k}$ for some $\lambda>1$, then

$$
\delta_{q_{k+1}}=\left\|\boldsymbol{R}\left(\boldsymbol{v}_{k+1}\right)\right\|=|\lambda|\left\|\boldsymbol{R}\left(\boldsymbol{v}_{k}\right)\right\|>\left\|\boldsymbol{R}\left(\boldsymbol{v}_{k}\right)\right\|=\delta_{q_{k}}
$$

a contradiction.

Proof of Theorem 3.5.

(i ) $\rightarrow$ (ii) This is Corollary 3.3.

(ii) $\rightarrow$ (i ) By (2.11) and the remark above for $k \geqq k_{0}$,

$$
\operatorname{dim}_{Q}\left(\boldsymbol{v}_{k}, \boldsymbol{v}_{k+1}\right)=\operatorname{dim}_{Q}\left(\boldsymbol{v}_{k}, \boldsymbol{v}_{k+1}, \boldsymbol{v}_{k+2}\right)=2 .
$$

Hence the $\boldsymbol{Q}$-vector space $W$ spanned by $\left[\boldsymbol{v}_{k+1}, \boldsymbol{v}_{k+2}\right]$ is a subspace of that spanned by $\left[\boldsymbol{v}_{k}, \boldsymbol{v}_{k+1}\right]$, so by dimension counting they are identical. Hence $W=\left[\boldsymbol{v}_{k}, \boldsymbol{v}_{k+1}\right]$ for all $k \geqq k_{0}$. Let $V$ denote the $\boldsymbol{Q}$-vector space orthogonal to $W$, of dimension $n-1$. Then $R=V \cap Z$ is a free $Z^{n-1}$-module of rank $n-1$, consisting of the $Z$-linear dependence relations satisfied by all $\boldsymbol{v}_{k}$ for $k \geqq k_{0}$. Then

$$
\frac{1}{q_{k}} \boldsymbol{v}_{k}=\left(1, \frac{p_{k, 1}}{q_{k}}, \cdots, \frac{p_{k, n}}{q_{k}}\right)
$$

satisfies all the relations of $R$. But

$$
\lim _{k \rightarrow \infty} \frac{1}{q_{k}} \boldsymbol{v}_{k}=\left(1, \alpha_{1}, \cdots, \alpha_{n}\right) .
$$

Consequently $\left[1, \alpha_{1}, \cdots, \alpha_{n}\right.$ ] also satisfies all the relations of $R$. Since $R$ has rank $n-1$,

$$
\operatorname{dim}_{Q}\left[1, \alpha_{1}, \cdots, \alpha_{n}\right] \leqq 2
$$

We next consider $Z$-linear dependencies among best approximation vectors $\alpha$ in the two-dimensional case. In order to prove Theorem 
1.1, we first prove the following lemma, wnich is analogous to Lemma 2.1 of Davenport and Schmidt [3]. (See also [2].)

Lemma 3.6. Let $\alpha \in \boldsymbol{R}^{2}-\boldsymbol{Q}^{2}$ and $\|\cdot\|$ be any norm on $\boldsymbol{R}^{2}$. There is a $k_{0}=k_{0}(\|\cdot\|)$ such that for $k \geqq k_{0}$ any two consecutive best approximation vectors $\boldsymbol{v}_{k}$ and $\boldsymbol{v}_{k+1}$ are a $\boldsymbol{Z}$-basis for the $\boldsymbol{Z}$-vectors in the $\mathbf{Q}$-vector space they span.

Proof. We may assume $\|\cdot\|$ is a scaled norm. Suppose $\boldsymbol{v}_{k}, \boldsymbol{v}_{k+1}$ are not a $Z$-basis, so there exist rational $\alpha, \beta$ not both integers such that

$$
\boldsymbol{w}=\left(w_{0}, w_{1}, w_{2}\right)=\alpha \boldsymbol{v}_{k}+\beta \boldsymbol{v}_{k+1}
$$

has $w \in Z^{3}$. By translation we may assume $|\alpha| \leqq 1 / 2,|\beta| \leqq 1 / 2$, not both zero. The denominator $w_{0}$ satisfies

$$
\left|w_{0}\right| \leqq|\alpha| q_{k}+|\beta| q_{k+1}<q_{k+1} .
$$

On the other hand by the triangle inequality

$$
\begin{aligned}
\|\boldsymbol{R}(\boldsymbol{w})\| & \leqq|\alpha|\left\|\boldsymbol{R}\left(\boldsymbol{v}_{k}\right)\right\|+|\beta|\left\|\boldsymbol{R}\left(\boldsymbol{v}_{k+1}\right)\right\| \\
& \leqq \frac{1}{2} \delta_{q_{k}}+\frac{1}{2} \delta_{q_{k+1}}<\delta_{q_{k}} .
\end{aligned}
$$

Now (3.13) and (3.14) contradict $\boldsymbol{v}_{k+1}$ being a best approximation vector, provided $\left|w_{0}\right| \neq 0$. Suppose $w_{0}=0$, so that $\boldsymbol{R}(\boldsymbol{w}) \in \boldsymbol{Z}^{n+1}$. By the remark after Theorem $3.5, \boldsymbol{v}_{k}$ and $\boldsymbol{v}_{k+1}$ are $\boldsymbol{R}$-linearly independent, hence $w \neq 0$ by (3.12). Since $\|\cdot\|$ is a scaled norm,

$$
\|\boldsymbol{R}(w)\| \geqq 1 \text {. }
$$

Now choose $k_{0}=k_{0}(\|\cdot\|)$ using Proposition 2.1 so that $\delta_{k}<1$ for all $k>k_{0}$. Then (3.14) and (3.15) contradict each other, completing the proof.

REMARK. For the sup norm $\|\cdot\|_{s}$ on $\boldsymbol{R}^{2}$ we can take $k_{0}\left(\|\cdot\|_{s}\right)=$ 1 in Lemma 3.6.

Proof of Theorem 1.1.

(ii) $\rightarrow$ (i ) Immediate, since rank $M_{k} \leqq 2$.

(i) $\rightarrow$ (ii) By the remark after Theorem 3.5, $v_{k}$ and $v_{k+1}$ are linearly independent over $\boldsymbol{Q}$, so $D_{k}=0$ implies $\boldsymbol{v}_{k+2}$ is a $\boldsymbol{Q}$-linear combination of $\boldsymbol{v}_{k+1}$ and $\boldsymbol{v}_{k}$. By Lemma 3.6, for $k \geqq k_{0}(\|\cdot\|)$ we can write $\boldsymbol{v}_{k+2}$ as a $Z$-linear combination

$$
\boldsymbol{v}_{k+2}=a \boldsymbol{v}_{k+1}+b \boldsymbol{v}_{k} \text {. }
$$


By the same reasoning $\boldsymbol{v}_{k}$ can be written as a $Z$-linear combination

$$
\boldsymbol{v}_{k}=c \boldsymbol{v}_{k+1}+d \boldsymbol{v}_{k+2} \text {. }
$$

Substituting this in (3.16) gives

$$
\boldsymbol{v}_{k+2}=(a+b c) \boldsymbol{v}_{k+1}+b d \boldsymbol{v}_{k+2} .
$$

Since $\boldsymbol{v}_{k+2}, \boldsymbol{v}_{k+1}$ are $Q$-linearly independent, this forces $b d=1$ so $b=$ \pm 1 and we have

$$
\boldsymbol{v}_{k+2}=a \boldsymbol{v}_{k+1} \pm \boldsymbol{v}_{k} .
$$

To complete the proof we must show

$$
\boldsymbol{v}_{k+2}=a \boldsymbol{v}_{k+1}-\boldsymbol{v}_{k}
$$

cannot occur. Suppose (3.20) were true. Then $q_{k+2}=a q_{k+1}-q_{k}>q_{k+1}$ so that $a \geqq 2$. Consider

$$
\boldsymbol{w}=\left(w_{0}, w_{1}, w_{2}\right)=\boldsymbol{v}_{k+1}-\boldsymbol{v}_{k} .
$$

The denominator $w_{0}$ satisfies

$$
0<w_{0}=q_{k+1}-q_{k}<q_{k+1} .
$$

Also

$$
\boldsymbol{w}=\frac{1}{a} \boldsymbol{v}_{k+2}-\left(1-\frac{1}{a}\right) \boldsymbol{v}_{k}
$$

Hence

$$
\begin{aligned}
\|\boldsymbol{R}(w)\| & \leqq \frac{1}{a}\left\|\boldsymbol{R}\left(\boldsymbol{v}_{k+2}\right)\right\|+\left(1-\frac{1}{a}\right)\left\|\boldsymbol{R}\left(\boldsymbol{v}_{k}\right)\right\| \\
& \leqq \frac{1}{a} \delta_{q_{k+2}}+\left(1-\frac{1}{a}\right) \delta_{q_{k}}<\delta_{q_{k}} .
\end{aligned}
$$

Then (3.21) and (3.23) contradict $\boldsymbol{v}_{k+1}$ being a best approximation vector.

4. Best approximation determinants. We give constructions of vectors exhibiting various pathological behaviors of the best approximation determinants, as described by Theorems 1.2 and 1.3.

Proof of Theorem 1.2. We will consider $\alpha=\left(\alpha_{1}, \cdots, \alpha_{n}\right)$ having the form

$$
\begin{aligned}
& \alpha_{1}=\sqrt{5} \\
& \alpha_{i}=\sqrt{5}+\sum_{j=1}^{\infty}\left(t_{i-1}\right)^{-f(j)} ; 2 \leqq i \leqq n
\end{aligned}
$$


where $t_{1}, \cdots, t_{n-1}$ are the first $n-1$ primes and $f(j)$ is an increasing integer-valued fuction satisfying

$$
\frac{f(j+1)}{f(j)} \geqq j \text { for all } j \geqq 1 \text {. }
$$

We show such $\alpha$ have the properties required by Theorem 1.3, provided $f(j)$ increases sufficiently rapidly.

We first check that (4.2) implies that $\operatorname{dim}_{Q}\left[1, \alpha_{1}, \cdots, \alpha_{n}\right]=n+1$. Suppose there were a linear dependence

$$
m_{0}+m_{1} \alpha_{1}+\cdots+m_{n} \alpha_{n}=0
$$

with integer $m_{i}$. We can rewrite this as $\beta_{1}=\beta_{2}$ where $\beta_{1}=m_{0}+$ $\left(m_{1}+\cdots+m_{n}\right) \sqrt{5}$ and

$$
\beta_{2}=-\sum_{i=2}^{n} m_{i} \sum_{j=1}^{\infty}\left(t_{i-1}\right)^{-f(j)} .
$$

Now $\beta_{1}$ is algebraic, while we claim $\beta_{2}$ is either a Liouville-type transcendental number or zero, the latter occurring only when $m_{2}=$ $\cdots=m_{n}=0$. Indeed, (4.4) clearly has a good rational approximation with denominator $\left(t_{1} \cdots t_{n-1}\right)^{f(j)}$. With the appropriate choice of numerator $H_{j}$, using (4.2) we have

$$
\left|\beta_{2}-\frac{H_{j}}{\left(t_{1} \cdots t_{n-1}\right)^{f(j)}}\right|<\frac{2 M}{t_{1}^{f(j+1)}}
$$

where $m=\operatorname{MAX}\left(\left|m_{i}\right|\right)$. Now write $t_{1} \cdots t_{n-1}=e^{c_{3}}$ and $t_{1}=e^{c_{4}}$. Then using (4.2),

$$
\left|\beta_{2}-\frac{H_{j}}{e^{c_{3} f(j)}}\right| \ll e^{-c_{1} j f(j)}
$$

This suffices to prove that $\beta_{2}$ is transcendental unless all $H_{j}=0$ from some point on. But $H_{j}=0$ gives the relation

$$
\sum_{j=2}^{N}\left[\frac{t_{1} \cdots t_{N}}{t_{j}}\right]^{f(j)} m_{j}=0
$$

Take a system of equations of type (4.5) for $N-1$ consecutive values of $j$. Viewed as linear equations in $m_{j}$ the determinant of this system is a Vandermonde determinant, and since all $\left(t_{1} \cdots t_{N}\right) / t_{j}$ are distinct it is nonzero. This requires that $m_{2}=m_{3}=\cdots=m_{N}=0$. Finally $\beta_{1}=m_{0}+m_{1} \sqrt{5}=0$ hence $m_{0}=m_{1}=0$.

The idea of the remainder of the proof is that the vector $\alpha$ quite closely approximates the vectors $\alpha^{(k)}=\left(\alpha_{1}^{(k)}, \cdots, \alpha_{n}^{(k)}\right)$ where 


$$
\begin{aligned}
& \alpha_{1}^{(k)}=\sqrt{5}, \\
& \alpha_{i}^{(k)}=\sqrt{5}+\sum_{j=1}^{k}\left(t_{i-1}\right)^{-f(j)} ; 2 \leqq i \leqq n .
\end{aligned}
$$

The vectors $\alpha^{(k)}$ have

$$
\operatorname{dim}_{Q}\left[1, \alpha_{1}^{(k)}, \cdots, \alpha_{n}^{(k)}\right]=2,
$$

and hence have all $D_{k}=0$ from some point on as described by Corollary 3.3. By choosing $f(j)$ to grow fast enough the behavior of the BSA's of $\alpha$ must mimic $\alpha^{(k)}$. The actual proof is complicated by the problem that we cannot guarantee that $\alpha$ has the same set of BSA's as $\alpha^{(k)}$ on any segment $k \leqq k_{0}$, no matter how close to $\alpha^{(k)}$ we make $\alpha$. This is because the $\alpha^{(k)}$ may be such that there is a $\operatorname{BSAD} q_{l}$ and a denominator $q^{\prime}$ with $q_{l+1}>q^{\prime}>q_{l}$ such that $\delta_{q_{l}}=\delta_{q^{\prime}}$. In this case an arbitrarily small perturbation of $\alpha^{(k)}$ to $\alpha$ may make $q^{\prime}$ a BSAD of $\alpha$. We circumvent this problem by proving that for sufficiently large $f(k+1)$ the BSA's of $\alpha$ will contain the BSA's of $\alpha^{(k)}$ on a long initial segment, and that new BSA's of $\alpha$ that are added in this initial segment will not affect a long chain of zero determinants. The first step is to analyze the behavior of the BSAD's $q_{m}=q_{m}^{(k)}$ to $\alpha^{(k)}$. We claim that

(i) There is a constant $C_{k}$ such that for all $m$,

$$
\delta_{q_{m}}<C_{k}\left(q_{m}\right)^{-1} \text {. }
$$

(ii) There is a constant $C_{k}^{\prime}$ such that for all $m$,

$$
q_{m+1}<C_{k}^{\prime} q_{m} .
$$

To show (i) we recall the one-diminsional BSAD's $q_{m}^{*}$ of $\sqrt{5}$ satisfy

$$
\left|q_{m}^{*} \sqrt{5}-p_{m}^{*}\right|<\left(q_{m}^{*}\right)^{-1} .
$$

Now consider the denominator

$$
\widetilde{q}=\left(t_{1} \cdots t_{n-1}\right)^{f(k)} q_{m}^{*} .
$$

Using (4.6) it is clear that we can choose numerators $\tilde{t}_{i}$ so that for all $i$,

$$
\left|\widetilde{q} \alpha_{i}^{(k)}-\widetilde{t}_{i}\right|<\left(t_{1} \cdots t_{n-1}\right)^{f(k)}\left(q_{m}^{*}\right)^{-1},
$$

that is (using (2.1))

$$
\delta_{\tilde{q}}<c_{2}\left(t_{1} \cdots t_{n-1}\right)^{2 f(k)}(\widetilde{q})^{-1} .
$$

On the other hand $\sqrt{5}$ is a badly approximable one-dimensional number (see $\S 5$ ). Using $\left|(p / q)^{2}-5\right| \geqq 1 / q^{2}$ we have 


$$
|q \sqrt{5}-p|>\frac{1}{3 \sqrt{5}} q^{-1}
$$

for all denominators $q$. This implies by (2.1) that for approximations to $\alpha^{(k)}$ we have

$$
\delta_{q}>\frac{1}{3} c_{1}(q)^{-1}
$$

Now (4.8) and (4.11) imply the BSAD's of $\sqrt{5}$ satisfy

$$
q_{m+1}^{*}<45 q_{m}^{*} \text {. }
$$

(Dirichlet's theorem would be violated otherwise.) Then by (4.9) for any $\operatorname{BSAD} q_{m}$ of $\alpha^{(k)}$ we can find $\widetilde{q}$ with

$$
\widetilde{q} \leqq q_{m} \leqq 45 \widetilde{q} \text {. }
$$

Then (4.10) implies (i) with

$$
C_{k}=45 c_{2}\left(t_{1} \cdots t_{n-1}\right)^{2 f(k)},
$$

since $\delta_{q_{m}}<\delta_{\tilde{q}}$. To prove (ii), given $q_{m}$ we can find $\widetilde{q}$ satisfying (4.10) with

$$
\frac{1}{45} C_{k}\left(\frac{1}{3} c_{1}\right)^{-1} q_{m}<\widetilde{q}<C_{k}\left(\frac{1}{3} C_{1}\right)^{-1} q_{m} .
$$

Then (4.9) and (4.10) give

$$
\delta_{\tilde{q}}<\frac{1}{3} c_{1}\left(q_{m}\right)^{-1}<\delta_{q_{m}} .
$$

This proves (ii) with

$$
C_{k}^{\prime}=C_{k}\left(\frac{1}{3} c_{1}\right)^{-1}
$$

We now inductively define the $f(k)$. The condition (4.2) implies that

$$
\left\|\alpha-\alpha^{(k)}\right\|<2 \cdot 2^{-f(k+1)} .
$$

Suppose $f(1), \cdots, f(k)$ are chosen. We will construct a block of at least $k$ consecutive zero best approximation determinants. First consider $\alpha^{(k)}$. All BSA's to $\alpha^{(k)}$ are $\left(c_{1}\right)^{-1}$-good approximations by (2.1). Consider those denominators $\widetilde{q}$ for $\alpha^{(k)}$ with

$$
\delta_{q_{m}}=\delta_{\tilde{q}} \text { and } q_{m}<\tilde{q}<q_{m+1} .
$$

Any such that occur are $\lambda_{k}$-good approximations with

$$
\lambda_{k}=\left(C_{k}^{\prime}\right)^{n}\left(c_{1}\right)^{-1},
$$


using (4.7). Now a set of $Z$-relations of $\operatorname{rank} n-1$ satisfied by the coordinates of $\alpha^{(k)}$ are

$$
\left(t_{i}\right)^{f(k)} \alpha_{i}^{(k)}-t_{i}^{f(k)} \alpha_{1}^{(k)}-\left[\sum_{j=1}^{k}\left(t_{i}\right)^{f(k)-f(j)}\right]=0
$$

for $2 \leqq i \leqq n$. The coefficients of these relations are bounded above by

$$
A_{k}=\left(t_{1} \cdots t_{n-1}\right)^{f(k)} .
$$

By Corollary 3.2 all $\lambda$-good approximations to $\alpha^{(k)}$ satisfy the relations (4.16) and hence lie in a certain 2-dimensional $\boldsymbol{Q}$-subspace $V_{k}$ provided that

$$
q>\left(n A_{k} \lambda_{k}\right)^{n}
$$

The following lemma guarantees that a suitable $f(k+1)$ can be chosen.

Lemma 4.1. Given any $\alpha \in \boldsymbol{R}^{n}$, any scaled $\|\cdot\|$ and any finite set $S$ of BSA's of $\alpha$ whose largest BSAD is $q_{L}$. Suppose $\delta_{q}<1 / 2$ for all $q$ in this set. Then there is an $\varepsilon=\varepsilon(\alpha, L)>0$ such that if $\alpha^{*}$ has

$$
\left|\alpha_{j}^{*}-\alpha_{j}\right|<\varepsilon
$$

for $1 \leqq j \leqq n$ then the BSA's in $S$ are also BSA's of $\alpha^{*}$.

Proof. The condition $\delta_{q}<1 / 2$ guarantees that the BSA's in $S$ are unique, by Lemma 2.2. Any BSA has the property $\delta_{q}(\alpha)<\delta_{q^{*}}(\alpha)$ for all $q^{*}<q$. This property for each pair $\left(q, q^{*}\right)$ is preserved for small perturbations of $\alpha$ since $\delta_{q}(\alpha)$ is a continuous function of $\alpha$. We can thus choose an $\varepsilon$ small enough to preserve this property for the finite set of pairs $\left(q, q^{*}\right)$ with $q^{*}, q \leqq q_{L}$ and $\delta_{q}(\alpha)<\delta_{q^{*}}(\alpha)$. This guarantees that when $\left|\alpha_{i}-\alpha_{i}^{*}\right|<\varepsilon$ for all $i$ then

$$
\delta_{q}(\alpha)<\delta_{q^{*}}(\alpha) \Longrightarrow \delta_{q}\left(\alpha^{*}\right)<\delta_{q^{*}}\left(\alpha^{*}\right),
$$

for all $q, q^{*} \leqq q_{L}$.

To complete the proof of Theorem 1.2, pick an index $L=L_{k}$ such that the BSA's of $\alpha^{(k)}$ satisfy

$$
q_{L}>\left(n A_{k} \lambda_{k}\right)^{n}
$$

and

$$
D_{L}=D_{L+1}=\cdots=D_{L+k}=0 .
$$


Pick an $\varepsilon=\varepsilon\left(\alpha^{(k)}, L_{k}\right)$ guaranteed to exist by Lemma 4.1 and choose $f(k+1)$ so large that

$$
2 \cdot 2^{-f(k+1)}<\varepsilon
$$

Then any $f(k+1)$ satisfying (4.20) and (4.2) has the properties

(i) The set of BSA's of $\alpha$ includes the BSA's $v_{m}$ of $\alpha^{(k)}$ with $L \leqq m \leqq L+k$.

(ii) Any other BSA's of $\alpha$ with BSAD $q$ satisfying $q_{L}^{(k)}<q<$ $q_{L+k}^{(k)}$ lie in the 2-dimensional $\boldsymbol{Q}$-subspace $V_{k}$.

Property (i) follows from Lemma 4.1, assuming $L$ is large enough that $\delta_{q}<1 / 2$. Property (ii) follows from the observation that (4.19) implies that any new BSAD's $q<q_{L+k}$ that occur for $\alpha$ must satisfy (4.15). But such approximations are $\lambda_{k}$-good by the choice of $\lambda_{k}$. Then (4.20) guarantees they lie in the subspace $V_{k}$.

Finally properties (i) and (ii) show that there is a block of at least $k+1$ consecutive BSA's of $\alpha$ lying in a 2 -dimensional $\boldsymbol{Q}$-subspace $V_{k}$, and hence giving at least $k$ consecutive zero best approximation determinants. Theorem 1.2 follows by induction on $k$.

Proof of Theorem 1.3. We will construct the desired vector $\alpha$ inductively as the limit of a sequence $\alpha^{(k)} \in \boldsymbol{R}^{2}$. All the $\alpha^{(k)}$ and $\alpha$ will sit in the unit square $I=[0,1] \times[0,1]$. In this construction we want $\alpha^{(k+1)}$ to leave unchanged a block of the first $L_{k}$ BSA's of $\alpha^{(k)}$. To this end we prove the following lemma.

LEMMA 4.2. The set $S$ of $\alpha$ in the unit square $I$ of $\boldsymbol{R}^{2}$ having the following two properties is dense.

(i) $\operatorname{dim}_{R}\left[1, \alpha_{1}, \alpha_{2}\right]=3$.

(ii) For the sup norm

$$
\delta_{q}(\alpha) \neq \delta_{q^{\prime}}(\alpha)
$$

whenever $q \neq q^{\prime}$.

Proof. It suffices to show $S$ has Lebesgue measure one, which implies denseness. In order for (i) to fail, there must be integers $m_{1}, m_{2}, m_{3}$ such that

$$
m_{1}+m_{2} \alpha_{1}+m_{3} \alpha_{2}=0 .
$$

In order for (ii) to fail, there must be integers $q_{1}, q_{2}^{\prime}, m_{4}, m_{5}$ with $q_{1} \neq q_{2}^{\prime}$ such that one of

$$
\begin{aligned}
& q_{1} \alpha_{1}+m_{4}=q_{2}^{\prime} \alpha_{1}+m_{5} \\
& q_{1} \alpha_{1}+m_{4}=q_{2}^{\prime} \alpha_{2}+m_{5} \\
& q_{1} \alpha_{2}+m_{4}=q_{2}^{\prime} \alpha_{2}+m_{5}
\end{aligned}
$$


holds. Each set $\left(m_{1}, m_{2}, m_{3}\right)$ or $\left(q_{1}, q_{2}^{\prime}, m_{4}, m_{5}\right)$ gives a constraint intersecting $I$ in a set of measure zero. There are only countably many such constraints, so the total removed has Lebesgue measure zero.

In the construction, we will choose each $\alpha^{(k)} \in S$. For any $\alpha \in S$ and any positive integer $L$, by Lemma 4.1 we can find an $\varepsilon=\varepsilon_{L}>0$ such that all vectors $\alpha^{*}$ in the sup norm open ball of radius $\varepsilon$, i.e.,

$$
\left\|\alpha-\alpha^{\prime}\right\|_{s}<\varepsilon
$$

all have the exactly same first $L$ BSAD's as $\alpha$. In this case (4.19) becomes for $q, q^{*}<q_{L}$ that

$$
\delta_{q}(\alpha)>\delta_{q^{*}}(\alpha) \Longleftrightarrow \delta_{q}\left(\alpha^{*}\right)>\delta_{q^{*}}\left(\alpha^{*}\right) .
$$

In the construction, we alternate back and forth between producing long blocks of zero determinants and large determinants. The zero determinants case is relatively easy and we merely sketch the proof. We first observe that the set

$$
T=\{(a+b \sqrt{5}, c+d \sqrt{5}) \mid a, b, c, d \in Z \text { and } b d \neq 0\}
$$

is dense in $I$. Suppose $\alpha_{2 k} \in S$ is constructed and the first $L_{2 k}$ BSA's are fixed. We pick a small open neighborhood $N_{2 k+1}$ of $\alpha_{2 k}$ whose closure $\bar{N}_{2 k+1}$ sits inside the previously constructed open neighborhood $N_{2 k}$, and which is so small that the first $L_{2 k}$ BSA's of any $\alpha^{\prime} \in N_{2 k+1}$ agree with $\alpha_{2 k}$. Since $T$ is dense, we can find a member $\alpha^{*}$ of $T$ in $N_{2 k}$. We choose $\alpha_{2 k+1}$ to be a member of $S$ sufficiently close to $\alpha^{*}$. Proceeding exactly as in the proof of Theorem 1.3, we can find a block of $N+2 k$ consecutive BSA's of $\alpha^{*}$ starting with $\boldsymbol{v}_{L}\left(\alpha^{*}\right)$ each of which sits in a 2-dimensional $Q$-subspace $V_{2 k}$, and such that all denominators $q^{\prime}$ with

$$
\delta_{q_{m}}=\delta_{q^{\prime}}, \quad q_{m}<q^{\prime}<q_{k+1}
$$

and $m \leqq L+2 k+2$ have associated approximation vectors $\boldsymbol{v}\left(q^{\prime}\right)$ lying in $V_{2 k}$. We can then show that any $\alpha$ sufficiently close to $\alpha^{*}$ will include all its BSA's $v_{m}$ with $L \leqq m \leqq L+2 k+2$ and possibly some other BSA's $v\left(q^{\prime}\right)$ from the set (4.22), and hence has a block of $\geqq 2 k$ consecutive zero determinants. Choosing $\alpha_{2 k+1} \in S$ to satisfy this, set $L_{2 k+1}=L^{*}$ where

$$
q_{L^{*}}\left(\alpha_{2 k+1}\right)=q_{L+2 k+2}\left(\alpha^{*}\right) .
$$

The interesting case is that of producing large determinants. Suppose $\alpha_{2 k+1} \in S$ and $L_{2 k+1}$ are given. Pick an open ball $N_{2 k+2}$ around $\alpha_{2 k+1}$ so small that all points $\alpha^{\prime}$ in it have the same first $L_{2 k+1}$ BSA's 
as $\alpha_{2 k+1}$ and so that $\bar{N}_{2 k+2} \subseteq N_{2 k+1}$. Since the set

$$
T^{*}=\left\{\left(\frac{P_{1}}{Q}, \frac{P_{2}}{Q}\right) \mid Q, P_{1}, P_{2} \in Z, Q \text { is prime, and } 0<P_{1}, P_{2}<Q\right\}
$$

is dense in $I$, we may choose $\beta_{0}=\left(P_{1} / Q, P_{2} / Q\right)$ from $T^{*}$ which lies in $N_{2 k+2}$. The set of BSA's of $\beta_{0}$ is finite since $\beta_{0} \in Q^{2}$. The first $L_{2 k+1}$ of these agree with those of $\alpha_{2 k+1}$, and the last one is $\left(Q, P_{1}, P_{2}\right)$. Of course

$$
\delta_{Q}\left(\beta_{0}\right)=0
$$

and $\delta_{q}\left(\beta_{0}\right) \neq 0$ for $0<q<Q$. Then

$$
\left|q \frac{P_{i}}{Q}-r\right| \geqq \frac{1}{Q} \quad \text { for } \quad i=1,2,
$$

when $1 \leqq q<Q$ and $r \in Z$, so that

$$
\delta_{q}\left(\beta_{0}\right) \geqq \frac{1}{Q}
$$

for all $q, Q$.

We next consider

$$
\beta_{1}=\left(\beta_{11}, \beta_{12}\right) \equiv\left(\frac{P_{1}}{Q}+\frac{1}{k_{1} Q}, \frac{P_{2}}{Q}+\frac{1}{k_{1} k_{2} Q}\right)
$$

where $k_{1}$ and $k_{2}$ will be large positive integers. We will show that if

$$
\begin{aligned}
& k_{1} \geqq 5 Q k_{2}, \\
& k_{2} \geqq Q^{3},
\end{aligned}
$$

then there is a determinant

$$
\left|D_{k}\left(\beta_{1}\right)\right| \geqq Q \text {. }
$$

To check this, we first claim that $\left(Q, P_{1}, P_{2}\right)$ is a BSA of $\beta_{1}$, so that there is an integer $l$ for which

$$
\boldsymbol{v}_{l+1}\left(\beta_{1}\right)=\left(Q, P_{1}, P_{2}\right) .
$$

Indeed using (4.25) we have

$$
\delta_{Q}\left(\beta_{1}\right)=\frac{1}{k}<\frac{1}{5}(Q)^{-4},
$$

while for $1 \leqq q \leqq Q$ we have (using (4.24))

$$
\begin{aligned}
\delta_{q}\left(\beta_{1}\right) & \geqq \delta_{q}\left(\beta_{0}\right)-\left\|q \beta_{1}-q \beta_{0}\right\|_{s} \\
& \geqq Q^{-1}-\frac{1}{5}(Q)^{-3},
\end{aligned}
$$


proving the claim.

Now we can write

$$
\boldsymbol{M}_{l}=\boldsymbol{M}_{l}\left(\beta_{1}\right)=\left[\begin{array}{l}
\boldsymbol{v}_{l} \\
\boldsymbol{v}_{l+1} \\
\boldsymbol{v}_{l+2}
\end{array}\right]=\left[\begin{array}{ccc}
q_{l} & p_{l, 1} & p_{l, 2} \\
Q & P_{1} & P_{2} \\
q_{l+1} & p_{l+1,1} & p_{l+1,2}
\end{array}\right] .
$$

Now suppose that there is a nonzero determinant $D_{l+m}=D_{l+m}\left(\beta_{1}\right)$ for some $m \geqq 0$. We will prove such an $m$ must exist later. Consider the least such $m$. Then by Theorem 1.2 there are positive integers $a_{j}$ such that

$$
\boldsymbol{v}_{l+j}=a_{j} \boldsymbol{v}_{l+j-1}+\boldsymbol{v}_{l+j-2}
$$

for $2 \leqq j \leqq m-1$. Hence

$$
\boldsymbol{M}_{l+m-2}\left(\beta_{1}\right)=\left[\begin{array}{l}
\boldsymbol{v}_{l+m-2} \\
\boldsymbol{v}_{l+m-1} \\
\boldsymbol{v}_{l+m}
\end{array}\right]=\boldsymbol{U T}
$$

where

$$
\boldsymbol{T}=\left[\begin{array}{ccc}
q_{l} & p_{l, 1} & p_{l, 2} \\
Q & P_{1} & P_{2} \\
q_{l+m} & p_{l+m, 1} & p_{l+m, 2}
\end{array}\right]
$$

and

$$
\boldsymbol{U}=\prod_{i=1}^{m-1} \boldsymbol{A}_{m-j}
$$

with

$$
\boldsymbol{A}_{j}=\left[\begin{array}{ccc}
0 & 1 & 0 \\
1 & a_{j} & 0 \\
0 & 0 & 1
\end{array}\right] \text { for } 1 \leqq j<m-1 .
$$

It is clear that $\operatorname{det} \boldsymbol{A}_{j}=-1, \operatorname{det} \boldsymbol{U}= \pm 1$. Hence

$$
|\operatorname{det} \boldsymbol{T}|=\left|\operatorname{det} \boldsymbol{M}_{l+m-2}\right|=\left|D_{l+m-2}\right| \neq 0 \text {. }
$$

Our goal is to show $|\operatorname{det} T| \geqq Q$.

Before doing this, we turn back to show the existence of an $m \geqq 0$ with $D_{l+m} \neq 0$. Suppose it did not exist. Now $\beta_{1} \in \boldsymbol{Q}^{2}$, so $\beta_{1}$ has a finite sequence of BSA's. Examining (4.25), we see that the last one is clearly

$$
\boldsymbol{v}_{l+n}=\left(k_{1} k_{2} Q, k_{1} k_{2} P_{1}+k_{2}, k_{1} k_{2} P_{2}+1\right)
$$

where $n \geqq 2$ is the appropriate integer. By hypothesis $D_{l+n}=0$ and 
(4.31) holds for $2 \leqq j \leqq n$. Then the formulae (4.32)-(4.35) hold with $m=n$. Thus

$$
\left|D_{l+n}\right|=\left|\operatorname{det} T^{*}\right|
$$

where

$$
\boldsymbol{T}^{*}=\left[\begin{array}{ccc}
q_{l} & p_{l, 1} & p_{l, 2} \\
Q & P_{\mathbf{1}} & P_{2} \\
q_{l+n} & p_{l+n, 1} & p_{l+n, 2}
\end{array}\right]
$$

But subtracting $k_{1} k_{2}$ times the second row of $T^{*}$ from the third and using (4.37) gives

$$
\begin{aligned}
\operatorname{det} T^{*} & =\left|\begin{array}{ccc}
q_{1} & p_{l, 1} & p_{l, 2} \\
Q & P_{1} & P_{2} \\
0 & k_{2} & 1
\end{array}\right| \\
& =-k_{2}\left|\begin{array}{cc}
q_{l} & p_{l, 2} \\
Q & P_{2}
\end{array}\right|+\left|\begin{array}{cc}
q_{l} & p_{l, 1} \\
Q & P_{1}
\end{array}\right| .
\end{aligned}
$$

Since $Q$ is prime and $P_{2}, q_{l}<Q$ we have $Q \nmid q_{l} P_{2}$ so that

$$
\left|\begin{array}{cc}
q_{l} & p_{l, 2} \\
Q & P_{2}
\end{array}\right| \neq 0
$$

and the first term on the right of (4.40) is at least $Q^{3}$ in absolute value. The second determinant on the right is bounded in absolute value by $2 Q^{2}$, hence $\operatorname{det} T^{*} \neq 0$, so $D_{l+n} \neq 0$, the required contradiction.

To show $|\operatorname{det} \boldsymbol{T}| \geqq Q$ we use (4.36). By subtracting appropriate multiples of the second row from the first and third row of $\boldsymbol{T}$ in (4.33) we obtain

$$
\operatorname{det} \boldsymbol{T}=\left|\begin{array}{ccc}
0 & \delta_{1} & \delta_{2} \\
Q & P_{1} & P_{2} \\
0 & \frac{r_{1}}{Q} & \frac{r_{2}}{Q}
\end{array}\right|=r_{1} \delta_{2}-r_{2} \delta_{1},
$$

where

$$
\begin{gathered}
\delta_{i}=p_{l, i}-q_{l} \frac{P_{i}}{Q} ; i=1,2, \\
\frac{r_{i}}{Q}=p_{l+m, i}-q_{l+m} \frac{P_{i}}{Q} ; i=1,2 .
\end{gathered}
$$

Note the $r_{i}$ are integers. We first bound the $\delta_{i}$. We have 


$$
1 \geqq\left|\delta_{i}\right| \geqq \frac{1}{Q},
$$

where (4.24) gives the right hand side, and the left side follows from $p_{l, i}$ being the nearest integer to $q_{l}\left(P_{i} / Q\right)$.

The key part of the proof is the estimation of the $r_{i}$. We claim that $r_{1}=k_{2} r_{2}$ and that $r_{2} \neq 0$. If this is so, then using the integrality of $r_{2}$,

$$
\begin{aligned}
|\operatorname{det} \boldsymbol{T}| & \geqq\left|\boldsymbol{r}_{1} \delta_{2}\right|-\left|\boldsymbol{r}_{2} \delta_{1}\right| \\
& \geqq\left|\boldsymbol{r}_{2}\right|\left(k_{2}\left|\delta_{2}\right|-\left|\delta_{1}\right|\right) \\
& \geqq \frac{k_{2}}{Q}-1 \\
& \geqq 4 Q^{2},
\end{aligned}
$$

using (4.41) and (4.26). To prove the claim, we start from (4.25), and obtain

$$
\delta_{Q}=\frac{1}{k_{1}}
$$

Now let $q=q_{l+m}$ so that

$$
\delta_{q}<\delta_{Q}=\frac{1}{k_{1}}
$$

This certainly implies that

$$
\begin{aligned}
& \left|q \beta_{1,1}-p_{l+m, 1}\right|<\frac{2}{k_{1}}, \\
& \left|q \beta_{1,2}-p_{l+m, 2}\right|<\frac{2}{k_{1}} .
\end{aligned}
$$

Substituting the definition (4.25) of $\beta_{1}$ into (4.47) and using (4.43) yields

$$
\begin{gathered}
\left|\frac{q}{k_{1}}-r_{1}\right|<\frac{2 Q}{k_{1}}, \\
\left|\frac{q}{k_{1} k_{2}}-r_{2}\right|<\frac{2 Q}{k_{1}} .
\end{gathered}
$$

These two inequalities give

$$
\begin{aligned}
\left|\frac{r_{1}}{k_{2}}-r_{2}\right| & \leqq\left|\frac{r_{1}}{k_{2}}-\frac{q}{k_{1} k_{2}}\right|+\left|\frac{q}{k_{1} k_{2}}-r_{2}\right| \\
& <\frac{2 Q}{k_{1} k_{2}}+\frac{2 Q}{k_{1}}
\end{aligned}
$$


Then (4.26) gives further

$$
\left|\frac{r_{1}}{k_{2}}-r_{2}\right|<\frac{2}{5 k_{2}^{2}}+\frac{2}{5 k_{2}}<\frac{1}{k_{2}} .
$$

But $r_{1}$ and $r_{2}$ are both integers, so $(4.50)$ forces $r_{1} \equiv 0\left(\bmod k_{2}\right)$ and then

$$
r_{1}=k_{2} r_{2} .
$$

Now $r_{2}=0$ gives $r_{1}=0$ which makes the bottom row of $T$ in (4.41) identically zero so $\operatorname{det} T=0$, contradicting (4.36). Hence $\left|r_{2}\right| \geqq 1$, proving the claim.

We now make the important observation that the argument above actually shows the stronger result that any approximation $\boldsymbol{v}=\boldsymbol{v}_{q}\left(\beta_{1}\right)$ with $\delta_{q}\left(\beta_{1}\right)<2 / k$ and with

$$
D(\boldsymbol{v})=\left|\begin{array}{c}
\boldsymbol{v}_{l}\left(\beta_{1}\right) \\
\boldsymbol{v}_{l+1}\left(\beta_{1}\right) \\
\boldsymbol{v}
\end{array}\right| \neq 0
$$

must have $D(\boldsymbol{v})>4 Q^{2}$.

To complete the proof, we now choose $k_{1}$ and $k_{2}$ satisfying (4.26) and (4.27) to be so large that $\beta_{1} \in N_{2 k+2}$. Then any $\alpha \in S$ sufficiently near $\beta_{1}$ will have the desired property. First we can guarantee $\alpha \epsilon$ $N_{2 k+2}$. Second, if $\alpha$ is close enough to $\beta_{1}$, by Lemma 4.1 we can guarantee it includes all the BSA's $v_{j}$ of $\beta_{1}$ with $q_{j} \leqq k_{1} k_{2} Q$. Now it may contain some extra BSA's $\boldsymbol{v}$ with $q<q_{l+m}$. Since $\boldsymbol{v}_{l+m}\left(\beta_{1}\right)$ is $\boldsymbol{Z}$-independent of $\boldsymbol{v}_{l}\left(\beta_{1}\right)$ and $\boldsymbol{v}_{l+1}\left(\beta_{1}\right)$, there will be some $k$ with

$$
D_{k}(\alpha)=\left|\begin{array}{c}
\boldsymbol{v}_{k}(\alpha) \\
\boldsymbol{v}_{k+1}(\alpha) \\
\boldsymbol{v}_{k+2}(\alpha)
\end{array}\right| \neq 0
$$

where

$$
q_{k+2}(\alpha) \leqq q_{l+m}\left(\beta_{1}\right) .
$$

We have now for a small $\varepsilon$,

$$
\delta_{Q}(\alpha)<\frac{1}{k}+\varepsilon
$$

so for $q=q_{k+2}(\alpha)$ also

$$
\delta_{q}(\alpha)<\frac{1}{k}+\varepsilon .
$$

For $\alpha$ sufficiently close to $\beta_{1}$, this implies 


$$
\delta_{q}\left(\beta_{1}\right)<\frac{2}{k}
$$

Then $D_{k}(\alpha) \equiv D(v)$ in (4.52) and so $D(v)>4 Q^{2}$. Hence we may choose $\alpha_{2 k+2}=\alpha$, and $L_{2 k+2}$ equal to that $L$ for which $q_{L}(\alpha)=k_{1} k_{2} Q$.

We are done by induction on $k$, since the $\alpha$ tend to a limit $\alpha$. The initial $L_{k}$ BSA's of $\alpha$ agree with those of $\alpha_{k}$ because $\alpha \in N_{k}$ for all $k$.

5. Badly approximable vectors. We recall that a vector $\alpha$ in $\boldsymbol{R}^{N}$ is said to be badly approximable with approximation constant $C$ if there are only finitely many solutions to the inequality

$$
\underset{1 \leqq i \leqq N}{\operatorname{MAX}}\left|q \alpha_{i}-p_{i}\right|<C|q|^{-1 / N} \text {. }
$$

By Dirichlet's theorem $0<C<1$.

This section shows that the best approximation vectors of badly approximable vectors $\alpha$ cannot behave as pathologically as the examples constructed in $\S 4$. Throughout this section we abbreviate $\delta_{q_{k}}$ to $\delta_{k}$.

THeOREM 5.1. Let $\alpha \in \boldsymbol{R}^{n}-\boldsymbol{Q}^{n}$ be a badly approximable vector, with approximation constant $C$. For a given norm $\|\cdot\|$ on $\boldsymbol{R}^{n}$ there is a constant $\Delta_{0}$ depending on $\|\cdot\|$ such that there are only finitely many $k$ for which

$$
\left|D_{k}\right|>\Delta_{0}(C)^{-n^{2}}
$$

Proof. The basic principle used is that the best approximation denominators with respect to a given norm $\|\cdot\|$ cannot be too far apart or Dirichlet's theorem will be violated. Suppose $\alpha$ is badly approximable with approximation constant $C$ and that (5.1) has no solutions for $q>Q_{0}$. Let $q_{k}>Q_{0}$ be a sup norm best approximation. Then

$$
\left(q_{k}\right)^{-1 / n} \geqq \delta_{k} \geqq C\left(q_{k}\right)^{-1 / n}
$$

By Dirichlet's theorem using (2.3) applied with $q=q_{k+1}-1$ gives

$$
\delta_{k} \leqq\left(q_{k+1}-1\right)^{-1 / n} .
$$

Combining this with (5.3) yields the bound

$$
q_{k+1}-1 \leqq C^{-n} q_{k} .
$$

Using (2.1) we derive analogous inequalities for the best approximations $q_{k}$ in another norm $\|\cdot\|$, which state that 


$$
\begin{aligned}
c_{2}\left(q_{k}\right)^{-1 / n} & \geqq \delta_{k} \geqq C c_{1}\left(q_{k}\right)^{-1 / n} \\
\delta_{k} & \leqq c_{2}\left(q_{k+1}-1\right)^{-1 / n} \\
q_{k+1}-1 & \leqq\left(\frac{c_{2}}{c_{1}}\right)^{n} C^{-n} q_{k} .
\end{aligned}
$$

Now consider for the approximations $q_{k}$ with respect to the general norm $\|\cdot\|$ the determinant

$$
D_{k}=\left[\begin{array}{cccc}
q_{k} & p_{k, 1} & \cdots & p_{k, n} \\
q_{k+1} & p_{k+1,1} & & p_{k+1, n} \\
\vdots & \vdots & & \vdots \\
q_{k+n} & p_{k+n, 1} & \cdots & p_{k+n, n}
\end{array}\right] .
$$

Subtract $\alpha_{i}$ times the first column from the $(i+1)^{s t}$ column, for $1 \leqq i \leqq n$. This leaves the determinant unchanged, and yields

$$
D_{k}=\left|\begin{array}{cccc}
q_{k} & -\xi_{k, 1} & \cdots & -\xi_{k, n} \\
\vdots & \vdots & & \vdots \\
q_{k+n} & -\xi_{k+n, 1} & \cdots & -\xi_{k+n, n}
\end{array}\right|
$$

where

$$
\xi_{i, m}=q_{i} \alpha_{m}-p_{i, m} .
$$

Note $\xi_{i, m}$ is the $m$ th entry in $\boldsymbol{R}\left(q_{i}\right)$, so that

$$
\left|\xi_{i, m}\right|<\delta_{l} \leqq \delta_{k} .
$$

Estimating (5.8) by absolute value estimates we obtain

$$
\begin{aligned}
\left|D_{k}\right| & \leqq n ! q_{k+n}\left(\delta_{k}\right)^{n} \\
& \leqq n !\left(c_{2}\right)^{n} q_{k+n}\left(q_{k}\right)^{-1},
\end{aligned}
$$

using (5.6), (5.11). Repeated use of (5.7) with $C_{0}=\left(c_{1} / c_{2}\right) C$ gives

$$
\begin{aligned}
q_{k+n} & \leqq C_{0}^{-n^{2}} q_{k}+1+C_{0}^{-n}+\cdots+C_{0}^{-n(n-1)} \\
& \leqq C_{0}^{-n^{2}}\left(q_{k}+n\right) .
\end{aligned}
$$

Applying this to (5.12) when $q_{k}>\operatorname{MAX}\left(Q_{0}, n\right)$ we obtain

$$
\left|D_{k}\right| \leqq \Delta_{0} C^{-n^{2}}
$$

where

$$
\Delta_{0}=2\left(c_{2}\right)^{n}\left(\frac{c_{1}}{c_{2}}\right)^{-n^{2}} n !
$$

depends only on $\|\cdot\|$. 
We next bound the number of consecutive $D_{k}=0$ that can occur in the two-dimensional case.

THEOREM 5.2. Let $\alpha \in \boldsymbol{R}^{2}-\boldsymbol{Q}^{2}$ be a badly approximable vector, with approximation constant $C$. For a given norm $\|\cdot\|$ on $\boldsymbol{R}^{2}$ there are constants $\Delta_{1}, \Delta_{2}$ depending on $\|\cdot\|$ such that there are only finitely many $k$ for which

$$
D_{k}=D_{k+1}=\cdots=D_{k+L}=0
$$

where

$$
L=\left[\Delta_{1}+\Delta_{2}|\log C|\right] .
$$

Proof. By Theorem $1.1 D_{k}=0$ for a given $k \geqq k_{0}(\|\cdot\|)$ implies that

$$
\boldsymbol{v}_{k}=a \boldsymbol{v}_{k-1}+\boldsymbol{v}_{k-2},
$$

for some positive integer $a$. In what follows we assume that $k \geqq$ $k_{0}(\|\cdot\|)$ satisfies (5.15) and (5.16). From this hypothesis we derive a contradiction, proving the theorem. By (5.17) we may write

$$
\boldsymbol{v}_{k+j}=a_{\jmath} \boldsymbol{v}_{k+j-1}+\boldsymbol{v}_{k+j-2}
$$

for $0 \leqq j \leqq L$, where the $a_{j}$ are positive integers.

We examine to what extent this mimicry (5.18) of the continued fraction algorithm carries over to the components of the approximation remainder vectors $\boldsymbol{R}\left(q_{k+j}\right)$. We use the notation

$$
\boldsymbol{R}\left(q_{l}\right)=\left(q_{l} \alpha_{1}-p_{l, 1}, q_{l} \alpha_{2}-p_{l, 2}\right) \equiv\left(\xi_{l, 1}, \xi_{l, 2}\right) .
$$

Then (5.18) implies

$$
\xi_{l, i}=a_{l \xi_{l-1, i}}+\xi_{l-2, i}
$$

for $l=k, k+1, \cdots, k+L$ and $i=1,2$. We shall call $\xi_{l, i}$ OCF-like if in the relation (5.20) we have

(i) $0<\left|\xi_{l, i}\right|<\left|\xi_{l-1, i}\right|$,

(5.22) (ii) $\xi_{l-1, i}$ and $\xi_{l, i}$ have opposite signs.

Otherwise we shall call $\xi_{l, i}$ non-OCF-like. The key facts we need are the following.

Fact 5.3. If $\xi_{l, i}$ is non-OCF-like, then

$$
\left|\xi_{l+j+1, i}\right| \geqq\left|\xi_{l+j, i}\right|
$$

for all $j \geqq 1$ for which (5.20) holds. 
Proof of Fact 5.3. If (ii) fails, then $\xi_{l-1, i}$ and $\xi_{l, i}$ have the same sign, or one of them is zero. In that case for all $l+j$ with $j \geqq 1$ the two terms on the right side of (5.20) have the same sign, so (5.23) holds for $j \geqq 0$. If (i) fails then (5.20) applied with $l+1$ shows $\xi_{l+1, i}$ and $\xi_{l, i}$ have the same sign, and as before (5.23) holds for $j \geqq 1$.

Fact 5.4. There is a value of $i=1,2$ and $m=0,1,2$ or 3 , such that the following both hold.

(i ) $\delta_{k+L-m}=\left|\xi_{k+L-m, i}\right|$

(ii) $\xi_{k+j, i}$ is OCF-like for $0 \leqq j \leqq L-m$.

Proof of Fact 5.4. Pick $i^{+}$such that

$$
\delta_{k+L-1}=\left|\xi_{k+L-1, i}\right| \text {. }
$$

If $\xi_{k+j, i}$ is not OCF-like for some $j$ with $j \leqq L-2$ then by Fact 5.3

$$
\left|\xi_{k+L, i}\right| \geqq\left|\xi_{k+L-1, i+}\right|=\delta_{k+L-1},
$$

which contradicts

$$
\delta_{k+L-1}>\delta_{k+L} \geqq\left|\xi_{k+L, i}\right| .
$$

Hence $\xi_{k+j, i^{+}}$is OCF-like for $0 \leqq j \leqq L-2$. If $\xi_{k+j, i}$ is OCF-like for $j=L-1$ we are done with $m=1, i=i^{+}$, so suppose not. We must also have

$$
\left|\xi_{k+L-1, i}\right| \geqq\left|\xi_{k+L-2, i}\right|
$$

in this case, otherwise (5.23) would hold $m=k+L-1$ and the proof of Fact 5.3 yields the same contradiction (5.26). Let $i^{-} \neq i^{+}$ denote the other value of $i$. Then (5.27) forces

$$
\delta_{k+L-2}=\left|\xi_{k+L-2, i^{-}}\right|>\left|\xi_{k+L-2, i}\right| \text {. }
$$

If $\xi_{k+j, i^{-}}$is non-OCF-like for some $j$ with $j \leqq L-3$ then by Fact 5.3

$$
\delta_{k+L-1} \geqq\left|\xi_{k+L-1, i}\right| \geqq\left|\xi_{k+L-2, i^{-}}\right|=\delta_{k+L-2},
$$

a contradiction. Hence $\xi_{k+j, i^{-}}$is OCF-like for $0 \leqq j \leqq L-3$ in this case. Now choose $m=3$ and $i$ to make (5.24) true.

We continue the proof of Theorem 5.2. Recall that the ordinary continued fraction (OCF) expansion of a number $\theta_{0}$ proceeds with partial quotients $q_{k}$ and partial remainders $\theta_{k}$ satisfying the recursion (for nonintegral $\theta_{k}$ ) 


$$
\theta_{k}=a_{k}+\frac{1}{\theta_{k+1}}
$$

where $\theta_{k}>1$ for $k \geqq 1$ and

$$
\theta_{k}-1<a_{k}<\theta_{k}
$$

for all $k$. Let

$$
\frac{P_{k}}{Q_{k}}=\left[a_{0}, a_{1}, \cdots, a_{k}\right]
$$

denote the kth convergent to $\theta_{0}$. Two well-known properties of the continued fraction algorithm which we will use are

$$
\left|\theta_{0}-\frac{P_{k}}{Q_{k}}\right|<\frac{1}{Q_{k}^{2}}
$$

and

$$
Q_{k}>\left(\frac{3}{2}\right)^{k-2}
$$

for all $k \geqq 1$.

Consider those values $L^{*}=L-j$ and $i$ for which Fact 5.4 holds. Since each $\xi_{k+j, i}$ is OCF-like for $0 \leqq j \leqq L^{*}$, each $\xi_{k+j, i}$ is nonzero. We define

$$
\theta_{j}=-\frac{\xi_{k+j-2, i}}{\xi_{k+j-1, i}}
$$

for $0 \leqq j \leqq L^{*}$. Dividing (5.20) by $\xi_{k+j-1}$ and rearranging yields

$$
\theta_{j}=a_{j}+\frac{1}{\theta_{j+1}}
$$

The conditions (5.21), (5.22) of being OCF-like show that

$$
\theta_{j} \geqq 1 \text { for } 0 \leqq j \leqq L^{*},
$$

and with (5.34) this implies

$$
\theta_{j}-1<a_{j}<\theta_{j} \text { for } 0 \leqq j \leqq L^{*} .
$$

Hence the $a_{k}$ 's of (5.20) agree with the first $L^{*}+1$ OCF partial quotients to $\theta_{0}$.

Now we can apply (5.31), (5.32) to derive the final contradiction. We obtain

$$
\left|\theta_{0}-\frac{P_{L^{*}}}{Q_{L^{*}}}\right|<\frac{1}{Q_{L^{*}}^{2}}
$$


The definition (5.33) of $\theta_{0}$ then shows that

$$
\begin{aligned}
\left|Q_{L^{*}} \xi_{k-2, i}+P_{L^{*}} \xi_{k-1, i}\right| & <\left(Q_{L^{*}}\right)^{-1}\left|\xi_{k-1, i}\right| \\
& <\left(Q_{L^{*}}\right)^{-1} \delta_{k-1} .
\end{aligned}
$$

On the other hand, the identity (5.20) and the continued fraction recurrence easily lead to

$$
\xi_{k+L^{*}, i}=P_{L^{*} \xi_{k-1, i}}+Q_{L^{*} \xi_{k-2, i}} .
$$

Using (5.24) and (5.36) we obtain

$$
\delta_{k+L^{*}}<\left(Q_{L^{*}}\right)^{-1} \delta_{k-1} .
$$

This will turn out to be too small to be compatible with the constraint (5.6), which asserts that

$$
\delta_{k+L^{*}}>C c_{1}\left(q_{k+L^{*}}\right)^{-1 / 2},
$$

since $\alpha$ is a $C$-badly approximable vector. Indeed by (5.18)

$$
q_{k+L^{*}}=P_{L^{*}} q_{k-1}+Q_{L^{*}} q_{k-2}
$$

in a similar way to (5.37). The bound

$$
P_{L^{*}} \leqq\left(q_{0}+2\right) Q_{L^{*}} q_{k-1}
$$

for continued fraction convergents is easy to establish by induction. We then obtain from (5.40) the bound

$$
q_{k+L^{*}} \leqq\left(a_{0}+2\right) Q_{L^{*}} q_{k+1} .
$$

We can bound $a_{0}$ by observing that for the BSAD's

$$
q_{k}=a_{0} q_{k-1}+q_{k-2}>a_{0} q_{k-1} .
$$

To avoid contradicting (5.7) we must have

$$
a_{0}<\left(\frac{c_{2}}{c_{1}}\right)^{2} C^{-2},
$$

where $c_{2}$ and $c_{1}$ are constants associated to the norm $\|\cdot\|$ by (2.1). So (5.41) becomes

$$
q_{k+L^{*}} \leqq\left(\left(\frac{c_{2}}{c_{1}}\right) C^{-2}+2\right) Q_{L^{*}} q_{k-1}
$$

and (5.39) becomes

$$
\delta_{k+L^{*}}>C\left(\left(\frac{c_{2}}{c_{1}}\right)^{2} C^{-2}+2\right)^{-1 / 2}\left(Q_{L^{*}}\right)^{-1 / 2}\left(q_{k-1}\right)^{-1 / 2} .
$$


Using (5.6) in (5.38) yields

$$
\delta_{k+L^{*}}<2 c_{2}\left(Q_{L^{*}}\right)^{-1}\left(q_{k-1}\right)^{-1 / 2} .
$$

These last two inequalities are contradictory whenever

$$
c_{2}<C\left(\left(\frac{2 c_{2}}{c_{1}}\right)^{2} C^{-2}+2\right)\left(Q_{L^{*}}\right)^{1 / 2},
$$

which is certainly true whenever

$$
Q_{L^{*}}>2 \frac{\left(c_{2}\right)^{4}}{\left(c_{1}\right)^{2}} \quad C^{-4}
$$

But by (5.32)

$$
Q_{L^{*}} \geqq Q_{L-3} \geqq\left(\frac{3}{2}\right)^{L-3} \text {. }
$$

Hence (5.48) shows that (5.47) holds whenever

$$
L \geqq \Delta_{1}+\Delta_{2}|\log C|,
$$

where

$$
\begin{gathered}
\Delta_{1}=\left(\log \frac{3}{2}\right)^{-1}\left(\log 2+4 \log c_{2}-2 \log c_{1}\right)+3, \\
\Delta_{2}=4\left(\log \frac{3}{2}\right)^{-1} .
\end{gathered}
$$

This completes the proof.

Proof of Theorem 1.4. For the sup norm $c_{1}=c_{2}=1$. The theorem then follows from Theorems 5.1 and 5.2 using (5.14), (5.49) and (5.50).

REMARK. For the special case of the sup norm on $R^{2}$, by taking more care in the estimates of Theorem 5.1 we can improve (i) of Theorem 1.4 to

$$
\left|D_{k}\right|>L \quad \text { with } \quad L=2+2 C^{-2}+2 C^{-4} .
$$

Using more detailed arguments involving the geometry of the sup norm, sharper bounds than (5.51) can be proved for badly approximable vectors with large approximation constants.

ACKNowledgments. I am indebted to W. W. Adams and F. Beukers, each of whom read a draft of this paper and suggested improvements. A. J. Brentjes brought my attention to the reference [7]. 


\section{REFERENCES}

1. A. J. Brentjes, Multi-dimensional Continued Fraction Algorithms, Math. Centre Tracts, Vol. 145, Mathematisch Centrum, Amsterdam, 1981.

2. H. Davenport and W. Schmidt, Approximation to real numbers by quadratic irrationals, Acta Arithmetica, 13 (1967), 169-176.

3. - A theorem on linear forms, Acta Arithmetica, 14 (1968), 209-223.

4. J. C. Lagarias, Some New Results in Simultaneous Diophantine Approximation, Proc. of the Queen's Number Theory Conference 1979 (P. Ribenboim, Ed.), Queen's Papers in Pure and Applied Math. No. 54 (1980), 453-474.

5. - Best simultaneous Diophantine approximations I. Growth rates of best approximation denominators, Trans. Amer. Math. Soc., in press.

6. S. Lang, Introduction to Diophantine Approximation, Addison-Wesley Publ. Co., Reading, Massachusetts, 1966.

7. J. T. Mack, A note on simultaneous approximation, Bull. Australian Math. Soc., 3 (1970), 81-83.

8. G. Szekeres, Multi-dimensional continued fractions, Ann. Univ. Sci. Budapest, Eötvös Sect. math., 13 (1970), 113-140.

Received July 15, 1980.

Bell Laboratories

Murray Hill, NJ 07974 


\section{PACIFIC JOURNAL OF MATHEMATICS}

\section{EDITORS}

DONALD BABBITT (Managing Editor)

University of California

Los Angeles, California 90024

HUGO ROSSI

University of Utah

Salt Lake City, UT 84112

C. C. MOORE and ARTHur AGUS

University of California

Berkeley, CA 94720
J. DUGUNDJI

Department of Mathematics

University of Southern California

Los Angeles, California 90007

R. FINN and J. MILGRAM

Stanford University

Stanford, California 94305

\section{ASSOCIATE EDITORS}
R. Arnes
E. F. BECKENBACH
B. H. NeumanN
F. WOLF
K. YösHIDA

\section{SUPPORTING INSTITUTIONS}

UNIVERSITY OF ARIZONA

UNIVERSITY OF BRITISH COLUMBIA

CALIFORNIA INSTITUTE OF TECHNOLOGY

UNIVERSITY OF CALIFORNIA

MONTANA STATE UNIVERSITY

UNIVERSITY OF NEVADA, RENO

NEW MEXICO STATE UNIVERSITY

OREGON STATE UNIVERSITY
UNIVERSITY OF OREGON

UNIVERSITY OF SOUTHERN CALIFORNIA

STANFORD UNIVERSITY

UNIVERSITY OF HAWAII

UNIVERSITY OF TOKYO

UNIVERSITY OF UTAH

WASHINGTON STATE UNIVERSITY

UNIVERSITY OF WASHINGTON 


\section{Pacific Journal of Mathematics}

Vol. 102, No. $1 \quad$ January, 1982

S. Agou, Degré minimum des polynômes $f\left(\sum_{i=0}^{m} a_{i} X^{p^{r i}}\right)$ sur les corps finis

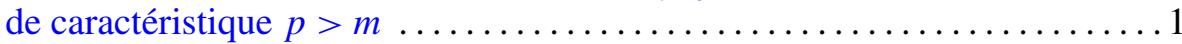

Chi Cheng Chen, On the image of the generalized Gauss map of a complete

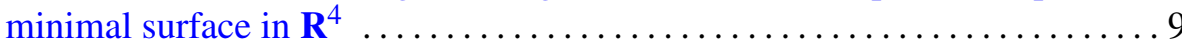

Thomas Curtis Craven and George Leslie Csordas, On the number of real

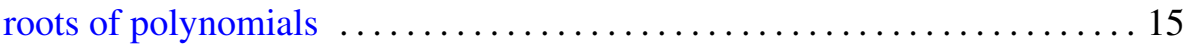

Allan L. Edelson and Kurt Kreith, Nonlinear relationships between oscillation and asymptotic behavior ....................... 29

B. Felzenszwalb and Antonio Giambruno, A commutativity theorem for

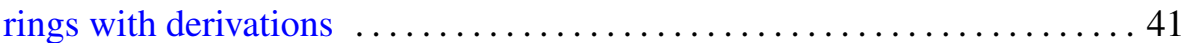

Richard Elam Heisey, Manifolds modelled on the direct limit of lines . . . . . 47

Steve J. Kaplan, Twisting to algebraically slice knots $\ldots \ldots \ldots \ldots \ldots \ldots 5$

Jeffrey C. Lagarias, Best simultaneous Diophantine approximations. II.

Behavior of consecutive best approximations $\ldots \ldots \ldots \ldots \ldots \ldots \ldots 61$

Masahiko Miyamoto, An affirmative answer to Glauberman's conjecture . . . 889

Thomas Bourque Muenzenberger, Raymond Earl Smithson and L. E.

Ward, Characterizations of arboroids and dendritic spaces ........... 107

William Leslie Pardon, The exact sequence of a localization for Witt

groups. II. Numerical invariants of odd-dimensional surgery

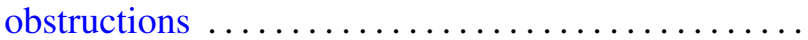

Bruce Eli Sagan, Bijective proofs of certain vector partition identities

Kichi-Suke Saito, Automorphisms and nonselfadjoint crossed products ...

John Joseph Sarraille, Module finiteness of low-dimensional PI rings ...

Gary Roy Spoar, Differentiable curves of cyclic order four . .

William Charles Waterhouse, Automorphisms of quotients of $\Pi \mathrm{GL}\left(n_{i}\right)$

Leslie Wilson, Mapgerms infinitely determined with respect to right-left equivalence

Rahman Mahmoud Younis, Interpolation in strongly logmodular

algebras 